\title{
A patient with osteoarthritis and cardiovascular disease presenting with bilateral hip pain: a case report Jennifer Yanow and Marco Pappagallo*
}

Address: Mount Sinai School of Medicine, Department of Anesthesiology, 1 Gustave L. Levy Place, New York, 10029 USA

Email: JY - jennifer.yanow@mssm.edu; MP* - marco.pappagallo@mssm.edu

* Corresponding author

Received: 30 July 2009 Accepted: II August 2009 Published: 24 August 2009

Cases Journal 2009, 2:8823 doi: 10.4076/1757-1626-2-8823

This article is available from: http://casesjournal.com/casesjournal/article/view/8823

(c) 2009 Yanow and Pappagallo; licensee Cases Network Ltd.

This is an Open Access article distributed under the terms of the Creative Commons Attribution License (http://creativecommons.org/licenses/by/3.0),

which permits unrestricted use, distribution, and reproduction in any medium, provided the original work is properly cited.

\begin{abstract}
Introduction: The pharmacological management of osteoarthritis normally begins with the administration of acetaminophen or a nonselective nonsteroidal anti-inflammatory drug. However, acetaminophen may not be efficacious in all patients, and nonsteroidal anti-inflammatory drugs may be associated with gastrointestinal and cardiovascular adverse effects.

Case presentation: A 79-year-old Caucasian man with bilateral hip pain was diagnosed with osteoarthritis of the hip. His past medical history included three prior myocardial infarctions, the most recent one occurring 2 years ago. He is taking aspirin $81 \mathrm{mg}$ once daily. Despite no history of ulcer disease or bleeding, he has been reluctant to take nonsteroidal anti-inflammatory drugs, and because of his cardiac history there is a contraindication to cyclooxygenase-2-specific inhibitors. He was started on naproxen $220 \mathrm{mg}$ twice daily, with the proton pump inhibitor omeprazole $20 \mathrm{mg}$ once daily. Upon follow-up at 4 weeks, his hip pain had decreased from a rating of 7 (on a ten-point scale) to 5 on his left side and from 5 to 2 on his right side. The patient began a course of physical therapy in conjunction with a regimen of naproxen $440 \mathrm{mg}$ in the morning and $220 \mathrm{mg}$ at night, plus the omeprazole and acetaminophen $650 \mathrm{mg}$ twice daily. He reported no gastrointestinal effects.

Conclusion: The addition of a proton pump inhibitor to nonsteroidal anti-inflammatory drug therapy can reduce the risk of peptic ulcer bleeding by $\geq 80 \%$, making the incidence of gastropathy the same as with cyclooxygenase-2-specific inhibitors. The fact that naproxen is not associated with an increased risk of acute myocardial infarction made it an appropriate choice for this patient.
\end{abstract}

\section{Introduction}

Osteoarthritis (OA) is the most common joint disorder in the United States and one of the leading causes of disability in the elderly. Approximately $5 \%$ of the population over the age of 65 years has radiographic evidence of OA of the hip, and nearly 200,000 total hip replacements are performed annually [1]. There is no cure for OA, but many pharmacologic options exist for managing its symptoms.

\section{Case presentation}

A 79-year-old Caucasian retired physician presented to the office with a chief complaint of bilateral hip pain that had been present for approximately 2 years but had become 
increasingly bothersome over the previous 3 months. He described the pain as dull and achy and located anteriorly in both hips with occasional radiation to the groin. At its most severe the pain was rated as a 7 on a ten-point scale in the left hip and as a 5 in the right hip. The pain was most severe in the morning, when the patient first got out of bed, and in the evening, after he had been active all day. There was no history of trauma, and the patient denied other joint symptoms. He reported that acetaminophen $650 \mathrm{mg}$ two or three times daily had been effective for managing his pain but no longer was. He tried ibuprofen $400 \mathrm{mg}$ three times daily, and while it provided adequate pain relief, he was reluctant to begin nonsteroidal antiinflammatory drug (NSAID) therapy for fear of gastrointestinal (GI) side effects. The patient had taken tramadol, hydrocodone, and oxycodone in the past but experienced troublesome lightheadedness.

The patient's past medical history was significant for coronary artery disease and three prior myocardial infarctions (MIs), the most recent having been 2 years earlier. He was told to take aspirin $81 \mathrm{mg}$ once daily to relieve his current pain. He denied any history of peptic ulcer disease, upper GI bleeding or other bleeding disorders, liver or kidney disease, chronic steroid use, and excessive alcohol intake.

On physical examination, the patient was able to walk without an assistive device but demonstrated a slightly antalgic gait. Hip internal rotation was painful and limited to $15^{\circ}$ bilaterally. External rotation was $50^{\circ}$ on the right side and $40^{\circ}$ on the left side. Hip flexion and extension were within normal limits. Flexion-abduction-external rotation (FABER) provocative testing produced hip pain bilaterally. Results of a lower-extremity strength examination are indicated in Table 1. Sensation was intact to light touch. Examination of the patient's knees demonstrated mild crepitus bilaterally, and results of a lumbar spinal examination were unremarkable.

This patient met the American College of Rheumatology clinical criteria for OA of the hip [2], which was hip pain plus hip internal rotation of $\geq 15^{\circ}$, pain with hip internal rotation, morning stiffness of the hip for $\leq 60$ minutes, and age $>50$ years or hip pain plus hip internal rotation of $<15^{\circ}$ and erythrocyte sedimentation rate (ESR) of $\leq 45 \mathrm{~mm} / \mathrm{hr}$ or hip flexion of $<115^{\circ}$ if the ESR is not known.

\section{Treatment}

The patient was concerned about long-term NSAID therapy because of potential GI side effects and was reluctant to retry opioid therapy since he had previously experienced lightheadedness. With the patient's prior history of MIs, cyclooxygenase (COX)-2-specific inhibitors were ruled out as a potential therapy. After reviewing the benefits and relative risk reduction of GI adverse events provided when a proton pump inhibitor (PPI) is used in combination with a nonselective NSAID, his physician decided to start the patient on over-the-counter (OTC) naproxen $220 \mathrm{mg}$ every 12 hours in conjunction with the PPI omeprazole $20 \mathrm{mg}$ once daily. The patient was advised of the black box warnings regarding naproxen that are related to cardiovascular (CV) and GI adverse events [3], as well as other possible side effects. He also advised the patient to start a program of physical therapy and instructed him to continue taking acetaminophen $650 \mathrm{mg}$ up to four times per day as needed.

When the patient returned to the office 4 weeks later, the patient had been in a physical therapy program for 3 weeks and was taking naproxen $440 \mathrm{mg}$ in the morning and $220 \mathrm{mg}$ at night, omeprazole $20 \mathrm{mg}$ once daily, and acetaminophen $650 \mathrm{mg}$ twice daily. He rated his pain on this regimen as a 5 on a ten-point scale in the left hip and a 2 in the right hip. He was given a straight cane by a physical therapist, which he found to be very helpful in alleviating some of his pain during walking. He reported no adverse GI effects and was pleased with his treatment and progress. He was able to tolerate physical therapy and was learning a home exercise program. A follow-up visit was scheduled for 2 months, during which time the patient will have completed his course of physical therapy; however, he will continue his home exercise program and adhere to his medication regimen.

\section{Discussion}

While initial treatment for OA should include nonpharmacologic intervention, when such therapy provides insufficient pain relief, medications should be added while the non-pharmacologic therapy is continued. After careful assessment of risk factors and medical co-morbidities, initial pharmacologic management should begin with acetaminophen or a nonselective NSAID, such as naproxen or ibuprofen. While the nonselective NSAIDs should be used with caution because of their potential for

Table I. Lower-Extremity Strength*

\begin{tabular}{|c|c|c|c|c|c|c|c|c|}
\hline & Hip Flexion & Hip Extension & Hip Abduction & Hip Adduction & Knee Extension & Knee Flexion & Ankle Dorsiflexion & Ankle Plantarflexion \\
\hline $\mathrm{R}$ & $5 / 5$ & $5 / 5$ & $4 / 5$ & $4+/ 5$ & $5 / 5$ & $5 / 5$ & $5 / 5$ & $5 / 5$ \\
\hline $\mathrm{L}$ & $5 / 5$ & $5 / 5$ & $4+/ 5$ & $4+/ 5$ & $5 / 5$ & $5 / 5$ & $5 / 5$ & $5 / 5$ \\
\hline
\end{tabular}

*Graded on a scale of I to 5 , where I represents minimal strength and 5 represents maximal strength. 
GI side effects, results from a recent observational study showed that the addition of a PPI to NSAID therapy reduced the risk of peptic ulcer bleeding by $82 \%$ [4]. The study also showed that patients taking a nonselective NSAID and a PPI have the same incidence of NSAIDinduced gastropathy as patients taking a COX-2-specific inhibitor. Currently, two fixed-dose combinations of an NSAID and a PPI are in development; naproxen/ esomeprazole from AstraZeneca [5] and ibuprofen/ famotidine from Horizon Therapeutics [6]. While both ibuprofen and naproxen are available in higher, prescription-strength doses as well as lower, OTC doses, the occurrence of adverse GI side effects in patients taking OTC doses of naproxen (up to $660 \mathrm{mg} /$ day) has been shown to be comparable to, and in some cases lower than, the incidence of GI side effects in patients taking placebo [7]. Additionally, while COX-2-specific inhibitors have been linked to an increased risk of CV events [8], naproxen has been shown not to be associated with an increased risk of acute MI $[9,10]$.

Another important issue that arises when initiating treatment for patients with OA who are already taking aspirin for cardioprotection is that certain NSAIDs may interfere with the antithrombotic benefits of aspirin through a competitive interaction with platelet COX-1. The co administration of ibuprofen and aspirin has been shown to antagonize aspirin's irreversible platelet inhibition, indicating that the use of ibuprofen in patients at increased CV risk could limit the cardioprotective effects of aspirin $[11,12]$. Post hoc analysis of the TARGET (Therapeutic Arthritis Research and Gastrointestinal Event Trial) study showed that among patients at high CV risk already taking aspirin, the incidence of CV events was significantly higher in the subgroup taking ibuprofen but not in the subgroup taking naproxen when each was compared with lumiracoxib [13]. The concomitant administration of acetaminophen $[11,14]$, or naproxen in prescription [15], or nonprescription doses [11], has not been shown to reduce the COX-1-mediated platelet inhibition induced by aspirin, suggesting that these two medications may be safer choices in patients at higher CV risk who are receiving aspirin therapy.

\section{Conclusions}

The application of clinical data to decision making is critical in determining the optimal treatment regimen for patients with OA. The use of OTC naproxen in association with a PPI and low-dose aspirin was found to be effective in treating OA of the hip in this patient with CV and GI risk factors.

\section{Abbreviations}

COX, cyclooxygenase; CV, cardiovascular; ESR, erythrocyte sedimentation rate; FABER, flexion-abduction- external rotation; GI, gastrointestinal; MI, myocardial infarction; NSAID, nonsteroidal anti-inflammatory drug; OA, osteoarthritis; OTC, over-the-counter; PPI, proton pump inhibitor; TARGET, Therapeutic Arthritis Research and Gastrointestinal Event Trial.

\section{Consent}

Written informed consent could not be obtained from the patient or the patient's next-of-kin because all parties were deceased/untraceable. We believe that patient cannot be identified from our manuscript and that this case report has a worthwhile clinical lesson/public health point.

\section{Competing interests}

The authors declare that they have no competing interests.

\section{Authors' contributions}

MP and JY contributed equally to the paper. MP edited and revised JY's original draft.

\section{References}

I. Lane N: Osteoarthritis of the hip. N Engl J Med 2007, 357:|4I3|42 I.

2. Altman R, Alarcón G, Appelrouth D, Bloch D, Borenstein D, Brandt K, Brown C, Cooke TD, Daniel W, Feldman D et al.: The American College of Rheumatology criteria for the classification and reporting of osteoarthritis of the hip. Arthritis Rheum 200I, 44:2455-2456.

3. Naproxen Black Box Warnings. [https://online.epocrates.com/ noFrame/].

4. Ray WA, Chung CP, Stein CM, Smalley WE, Hall K, Arbogast PG, Griffin MR: Risk of peptic ulcer hospitalizations in users of NSAIDs with gastroprotective cotherapy versus coxibs. Gastroenterology 2007, I33:790-798.

5. Product candidates. [http://www.pozen.com/product/pn.asp].

6. HZT-50I Phase 3 Enrollment Closed: Clinical Data Expected Fall 2008. [http://www.horizontherapeutics.com/wt/page/hzt_50I].

7. Bansal V, Dex T, Proskin H, Garreffa S: A look at the safety profile of over-the-counter naproxen sodium: a meta-analysis. J Clin Pharmacol 2001, 41:127-138.

8. Chou R, Helfand M, Peterson K, Dana T, Roberts C: Comparative Effectiveness and Safety of Analgesic for Osteoarthritis. Comparative Effectiveness Review No. 4. (Prepared by the Oregon Evidence-based Practice Center Under Contract No. 290-02-0024.) Rockville, MD: Agency for Healthcare Research and Quality. September 2006. [http://www.effectivehealthcare.ahrq.gov/reports/final.cfm] Accessed April 3, 2008.

9. Kearney PM, Baigent C, Godwin J, Halls H, Emberson JR, Patrono C: Do selective cyclo-oxygenase-2 inhibitors and traditional non-steroidal anti-inflammatory drugs increase the risk of atherothrombosis? Meta-analysis of randomised trials. BMJ 2006, 332:1302-I308.

10. McGettigan P, Henry D: Cardiovascular risk and inhibition of cyclooxygenase: a systematic review of the observational studies of selective and nonselective inhibitors of cyclooxygenase 2. JAMA 2006, 296:1633-1644.

II. Catella-Lawson F, Reilly MP, Kapoor SC, Cucchiara AJ, DeMarco S, Tournier B, Vyas SN, FitzGerald GA: Cyclooxygenase inhibitors and the antiplatelet effects of aspirin. N Engl J Med 200I, 345:1809-1817.

12. Advil® (ibuprofen) Product Information. [www.advil.com/ products/advil/tablet_label.asp].

13. Farkouh ME, Greenberg JD, Jeger RV, Ramanathan K, Verheugt FW, Chesebro JH, Kirshner H, Hochman JS, Lay CL, Ruland S, Mellein B, Matchaba PT, Fuster V, Abramson SB: Cardiovascular outcomes in high-risk patients with osteoarthritis treated with ibuprofen, naproxen, or lumiracoxib. Ann Rheum Dis 2007, 66:764-770. 
14. Brune K, Hochberg MC, Schiff M, Oldenhof J, Schuller R, Zlotnick S: The platelet inhibitory effects of the combination of naproxen sodium or acetaminophen with low-dose aspirin [abstract]. Arthritis Rheum 2007, 56:S359.

15. Capone ML, Tacconelli S, Sciulli MG, Grana M, Ricciotti E, Minuz P, Di Gregorio P, Merciaro G, Patrono C, Patrignani P: Clinical pharmacology of platelet, monocyte, and vascular cyclooxygenase inhibition by naproxen and low-dose aspirin in healthy subjects. Circulation 2004, 109:|468-|47|.

\section{Do you have a case to share?}

Submit your case report today

- Rapid peer review

- Fast publication

- PubMed indexing

- Inclusion in Cases Database

Any patient, any case, can teach us something

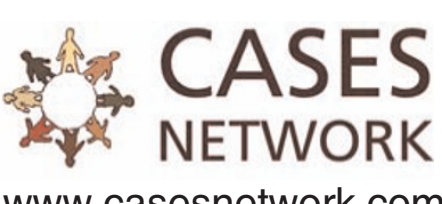

www.casesnetwork.com 